\title{
Doença Meningocócica Invasiva: Aplicação do Base Excess and Platelets Score numa Unidade de Cuidados Intensivos Pediátricos Portuguesa
}

\author{
Invasive Meningococcal Disease: Application of Base Excess and Platelets \\ Score in a Portuguese Paediatric Intensive Care Unit
}

\author{
Luís MARTINS ${ }^{1}$, Patrícia MAÇÃO ${ }^{1}$, Carla PINTO $\otimes^{1}$, Teresa DIONÍSIO'1, Andrea DIAS ${ }^{1}$, Alexandra DINIS ${ }^{1}$, Leonor \\ CARVALHO ${ }^{1}$, José Farela NEVES ${ }^{1}$ \\ Acta Med Port 2015 May-Jun;28(3):342-346
}

\section{RESUMO}

Introdução: A infeção meningocócica tem uma elevada mortalidade e morbilidade. Recentemente foi desenvolvido um score de prognóstico para a doença meningocócica invasiva em idade pediátrica, baseado na contagem plaquetar e no excesso de base - 0 Base Excess and Platelets Score. O objetivo principal desde estudo foi avaliar a precisão prognóstica do Base Excess and Platelets Score em doentes admitidos em cuidados intensivos pediátricos por doença meningocócica invasiva.

Material e Métodos: Estudo observacional, com colheita de dados retrospetiva, que incluiu um período de 13,5 anos (01/2000 a 06/2013). Foram analisados: mortalidade por doença meningocócica invasiva e fatores associados (disfunção de órgão e falência multi-órgão). Foi calculado o Base Excess and Platelets Score de forma retrospetiva, para avaliar a sua precisão na predição da mortalidade e foi comparado com o Paediatric Risk of Mortality e Paediatric Index of Mortality ${ }_{2}$.

Resultados: Foram admitidas 76 crianças com doença meningocócica invasiva. O tipo de disfunção mais frequente foi a cardiovascular (92\%), seguida da hematológica (55\%). Cumpriram critérios de falência multi-órgão 47 doentes (62\%). A mortalidade global foi de $16 \%$. A disfunção neurológica e a renal foram as que apresentaram uma maior associação com a mortalidade, odds ratio ajustado 315 (26 - 3 804) e 155 (20 - 1299). Após aplicação das curvas receiver operating characteristic, o Base Excess and Platelets Score tinha uma area under curve de 0,81, o Paediatric Index of Mortality ${ }_{2}$ de 0,91 e o Paediatric Risk of Mortality de 0,96.

Discussão: O Base Excess and Platelets Score apresentou uma boa precisão apesar de não tão elevada como o Paediatric Index of Mortality ${ }_{2}$ ou o Paediatric Risk of Mortality.

Conclusões: O Base Excess and Platelets Score pode ser útil como indicador prognóstico na doença meningocócica invasiva, por apresentar uma elevada sensibilidade e especificidade e ser objetivo e rapidamente disponível na admissão.

Palavras-chave: Criança; Cuidados Intensivos Pediátricos; Doença Meningocócica; Portugal; Prognóstico; Valor Preditivo dos Testes.

\section{ABSTRACT}

Introduction: Meningococcal infection has a high mortality and morbidity. Recently a new prognostic scoring system was developed for paediatric invasive meningococcal disease, based on platelet count and base excess - base excess and platelets score. The main objective of this study was to evaluate the accuracy of base excess and platelets score to predict mortality in children admitted to intensive care due to invasive meningococcal disease.

Material and Methods: Observational study, with retrospective data collection, during a 13.5 years period (01/2000 to 06/2013). Mortality by invasive meningococcal disease and related factors (organ dysfunction and multi-organ failure) were analysed. The base excess and platelets score was calculated retrospectively, to evaluate its accuracy in predicting mortality and compared with Paediatric Risk of Mortality and Paediatric Index of Mortality

Results: Were admitted 76 children with invasive meningococcal disease. The most frequent type of dysfunction was cardiovascular $(92 \%)$, followed by hematologic $(55 \%)$. Of the total, 47 patients $(62 \%)$ had criteria for multi-organ failure. The global mortality was $16 \%$. Neurologic and renal dysfunction showed the strongest association with mortality, adjusted odds ratio 315 (26 - 3804 ) and 155 (20 - 1299 ). After application of receiver operating characteristic curves, Base Excess and Platelets score had an area under curve of 0.81, Paediatric Index of Mortality ${ }_{2}$ of 0.91 and Paediatric Risk of Mortality of 0.96 .

Discussion: The Base Excess and Platelets score showed good accuracy, although not as high as Paediatric Risk of Mortality or Paediatric Index of Mortality

Conclusions: The Base Excess and Platelets score may be useful tool in invasive meningococcal disease because is highly sensitive and specific and is objectively measurable and readily available at presentation.

Keywords: Child; Intensive Care Units, Pediatric; Meningococcal Infections; Portugal; Predictive Value of Tests; Prognosis.

\section{INTRODUÇÃO}

A infeção meningocócica pode ter evolução rápida para sépsis, choque sético e falência multiorgânica. ${ }^{1,2}$

A elaboração de scores prognósticos tem dois objetivos principais: identificar os doentes com risco elevado de deterioração clínica rápida e estratificar o risco para ensaios de novos fármacos ou testes diagnósticos futuros. ${ }^{3-5}$
Existem scores de prognóstico, validados para doença meningocócica invasiva (DMI) que combinam dados clínicos e laboratoriais. Nestes estão incluídos o Glasgow Meningococcal Septicaemia Prognostic Score (GMSPS), ${ }^{6}$ e scores genéricos para cuidados intensivos pediátricos como o Paediatric Risk of Mortality $(\mathrm{PRISM})^{7}$ e o

1. Serviço de Cuidados Intensivos Pediátricos. Hospital Pediátrico. Centro Hospitalar e Universitário de Coimbra. Coimbra. Portugal.

$\bowtie$ Autor correspondente: Carla Pinto. carla.regina.pinto@gmail.com

Recebido: 29 de Agosto de 2014 - Aceite: 19 de Março de 2015 | Copyright (C) Ordem dos Médicos 2015 
Paediatric Index of Mortality ${ }_{2}\left(\mathrm{PIM}_{2}\right) \cdot{ }^{8}$ Existem ainda scores de prognóstico que se baseiam exclusivamente em dados laboratoriais, como o score de Roterdão. ${ }^{9}$

Um score ideal deve incluir um número mínimo de variáveis, que possam ser rápida e objetivamente mensuráveis, na apresentação da doença e ser custo-efetivas. ${ }^{3}$

É importante ter em conta que alguns scores, como o PRISM ou o GMSPS, usam dados clínicos, estando dependentes de uma avaliação que é subjetiva em muitos aspetos, o que pode reduzir a sua fiabilidade..$^{3,6,7}$

Recentemente foi desenvolvido e validado um novo score prognóstico por um grupo de investigadores de vários centros europeus de cuidados intensivos pediátricos, baseado na contagem plaquetar e no excesso de base, o BEP score (base excess and platelets). Este score é calculado pela fórmula: ${ }^{3}$

$1 /\left(1+\mathrm{e}^{\left(0,18909 \times \text { Excesso Base, mmo/L)+(0,01015 ×Plaquetas, } 10^{*} 12 / \mathrm{L}\right)+3,07861}\right)$

Assim, o objetivo principal deste trabalho foi avaliar a precisão do BEP score na predição da mortalidade na nossa população e compará-lo com outros scores usados em cuidados intensivos. Os objetivos secundários foram analisar a mortalidade por doença meningocócica invasiva e fatores associados.

\section{MATERIAL E MÉTODOS}

Foi efetuado um estudo observacional com colheita de dados retrospetiva num serviço de cuidados intensivos de um hospital pediátrico referência para a região centro do país.

Foram incluídas todas as crianças internadas com diagnóstico de DMI (definitivo ou provável) admitidas entre janeiro de 2000 e junho de 2013 (13,5 anos).

Definiu-se DMI de acordo com os critérios do Centers for Disease Control and Prevention de 2010. ${ }^{10}$

Foram colhidas as seguintes variáveis através da consulta dos registos do processo clínico e da base de dados do serviço: ano de admissão, idade, género, isolamento e serótipo do meningococo, tipo de disfunção de órgão, mortalidade e indicador de prognóstico (PRISM).

Foi calculado o BEP score de forma retrospetiva a todos os doentes em que havia avaliação com gasometria (quantificação do excesso de base) e hemograma (conta- gem plaquetar) realizados na primeira hora de admissão. $\mathrm{O} \mathrm{PIM}_{2}$ também foi calculado de forma retrospetiva nos anos anteriores aos da sua publicação.

A análise estatística dos dados foi efetuada com recurso ao Statistical Package for the Social Science ${ }^{\circledR}$, versão 20. A caracterização da população foi feita pelo cálculo de medidas de tendência central e de dispersão para variáveis quantitativas e pela determinação de frequências absolutas e relativas para variáveis qualitativas. Após aplicação de um teste de normalidade (Kolmogorov-Smirnov) verificou-se que as variáveis quantitativas não tinham distribuição normal, pelo que foram caracterizadas com a mediana e amplitude inter-quartil (AIQ). Na comparação de variáveis nominais e quantitativas sem distribuição normal foi utilizado o teste de Mann-Whitney. Para a comparação de variáveis nominais foram utilizados os testes de qui-quadrado ou exato de Fisher, de acordo com as regras de Cochran.

Foi utilizada a regressão logística para inferir a associação entre os diferentes tipos de falência de órgão e a mortalidade.

Foi considerado um nível de significância de $5 \%$.

Foram aplicadas curvas receiver operating characteristic (ROC) para o BEP score, PRISM e $\mathrm{PIM}_{2}$ e aplicada a fórmula de Youden para obter um cut-off que maximizasse a sensibilidade e especificidade em relação à mortalidade.

Este estudo obedeceu aos princípios éticos para investigação médica em seres humanos consagrada na Declaração de Helsínquia da Associação Médica Mundial.

\section{RESULTADOS}

Durante o período de estudo foram admitidas 76 crianças com diagnóstico de DMI, correspondentes a 1,6\% do total de admissões nos cuidados intensivos. A distribuição ao longo dos anos foi variável, com um mínimo de zero casos em 2011 e um máximo de 12 casos em 2002 (Fig. 1). A mediana da idade foi de 2,2 anos (AIQ: 0,8 - 4,5). Verificou-se um predomínio do género masculino (43/76, 56,6\%).

O meningococo foi isolado em cultura de líquidos biologicamente estéreis em $57,9 \%$ dos casos (44/76). Foi possível identificar o serogrupo da $N$. meningitidis em 21 casos $(47,2 \%)$, sendo mais frequente o serogrupo $B(12)$, seguido do $C$ (oito doentes) e do $Y$ (um doente). Desde 2005 que

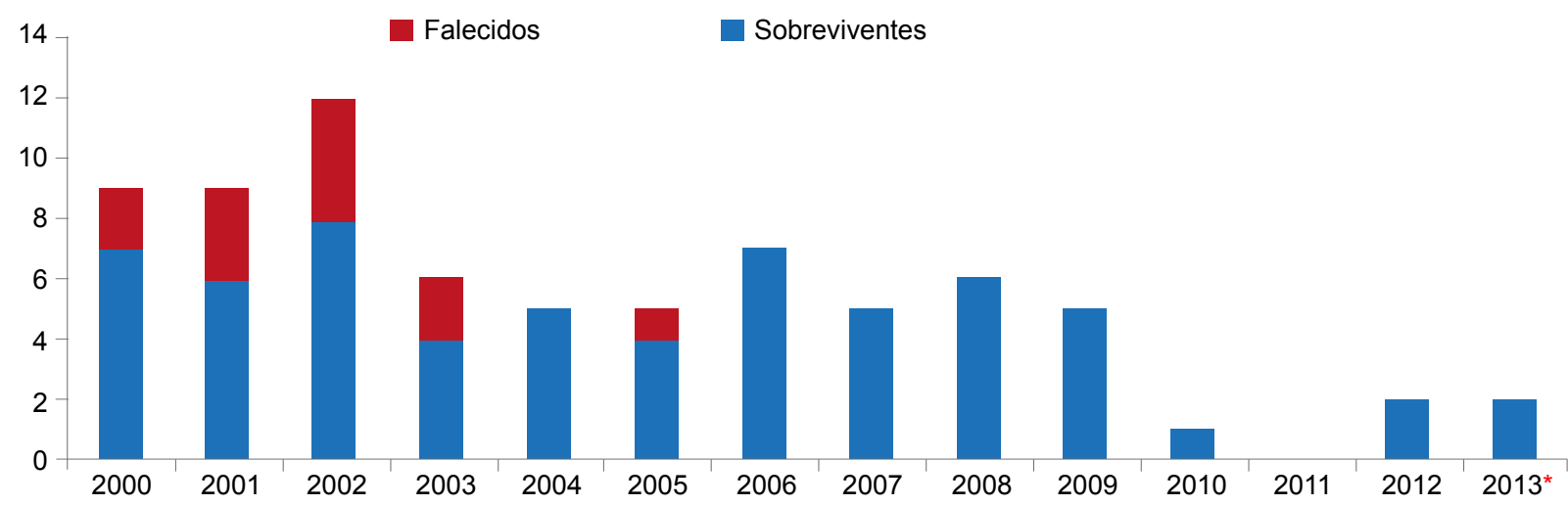

Figura 1 - Doentes admitidos em cuidados intensivos pediátricos por DMI $(n=76)$

* incluídos apenas 6 meses 
Tabela 1 - Tipos de disfunção de órgão nos falecidos e sobreviventes $(n=76)$

\begin{tabular}{|c|c|c|c|}
\hline Tipo de disfunção de órgão & $\begin{array}{l}\text { Falecidos } \\
(n=12)\end{array}$ & $\begin{array}{c}\text { Sobreviventes } \\
\qquad(n=64)\end{array}$ & $p$ \\
\hline Renal & $83,3 \%$ & $3,1 \%$ & $\begin{array}{c}<0,001 \\
(\text { Mann Whitney U) }\end{array}$ \\
\hline Hematológica & $91,7 \%$ & $48,4 \%$ & $\begin{array}{c}0,06 \\
\text { (Qui-quadrado) }\end{array}$ \\
\hline Neurológica & $83,3 \%$ & $1,6 \%$ & $\begin{array}{c}0,71 \\
\text { (Mann Whitney) }\end{array}$ \\
\hline Respiratória & $91,7 \%$ & $17,2 \%$ & $\begin{array}{c}<0,001 \\
\text { (Mann Whitney) }\end{array}$ \\
\hline Cardiovascular & $91,7 \%$ & $92,2 \%$ & $\begin{array}{c}1 \\
\text { (Mann Whitney) }\end{array}$ \\
\hline Falência multi-orgão & $100 \%$ & $54,7 \%$ & $\begin{array}{c}0,02 \\
\text { (Mann Whitney) }\end{array}$ \\
\hline
\end{tabular}

não há isolamento do serotipo $C$.

Clinicamente, a maioria dos doentes (50/76, 65,8\%) apresentou púrpura rapidamente progressiva e 40,8\% (31/76) meningite. Durante a evolução da infeção cumpriram critérios de falência multi-orgão 47 doentes $(61,8 \%)$. $\mathrm{O}$ tipo de disfunção mais frequente foi a cardiovascular (70/76, 92,1\%), seguido da disfunção hematológica (42/76, $55,3 \%)$ e respiratória $(22 / 76,28,9 \%)$. A maioria das crianças teve suporte cardiovascular $(71 / 76,93,4 \%)$ e algumas tiveram ventilação invasiva $(21 / 76,27,6 \%)$ e hemodiafiltração $(3 / 76,3,9 \%)$.

Nos 76 doentes incluídos no estudo, a mediana do PRISM score foi 3,2\% (AIQ: 1,1 - 20,4\%) e a do PIM $_{2}$ foi 1,1\% (AIQ: 0,9 - 3,6\%). Foi possível calcular o BEP score em 65 doentes $(85,5 \%)$. Nestes, a mediana foi 0,26 (AIQ: $0,06-0,72$ ).

A mortalidade global foi $15,8 \%$ (12/76).

A mediana da idade nos doentes falecidos foi 1,6 anos (AIQ: 0,8 - 2,6) e nos sobreviventes 2,4 anos (AIQ: 0,9 - 5,3; $p=0,176$, teste de Mann-Whitney).

A púrpura rapidamente progressiva ocorreu em 91,7\% dos falecidos e em $60,9 \%$ dos sobreviventes ( $p=0,049$; teste de Fisher).

Não foram encontradas diferenças significativas relativamente à presença de meningite (falecidos: $33,3 \%$ versus sobreviventes: $42,2 \% ; p=0,71$; teste de Fisher).

Relativamente à disfunção de órgão, tal como se pode observar na Tabela 1, todos os tipos foram mais frequentes nas crianças que faleceram com exceção da disfunção cardiovascular.

Após regressão logística, verificou-se que a falência de órgão mais fortemente associada à mortalidade foi a neurológica, seguida da renal (Tabela 2).

A mediana do PRISM nos doentes falecidos foi $52,4 \%$ (AIQ: 23,7 - 82,5) e nos sobreviventes foi 2,2\% (AIQ: 1,0 $10,1)(p<0,001$, teste de Mann-Whitney).

A mediana do $\mathrm{PIM}_{2}$ foi $13,3 \%$ (AIQ: 3,0 - 76,1) nos doentes falecidos e nos sobreviventes foi 1,1\% (AIQ: 0,9-2,3\%) $(p<0,001$, teste de Mann-Whitney).

A mediana do BEP score foi de 0,14 (AIQ: 0,21 - 0,69) nos doentes falecidos enquanto nos sobreviventes foi de 0,02 (AIQ: 0,01 - 0,04) ( $p=0,001$, teste de Mann-Whitney).

Aplicando as curvas ROC para o PRISM, obtivemos uma area under curve (AUC) de 0,96 (0,91 - 1,00). Através da fórmula de Youden a sensibilidade foi de $100 \%$ e a especificidade de $87,5 \%$ para um cut-off de $18,4 \%$. Para o $\mathrm{PIM}_{2}$, obtivemos uma AUC de 0,91 (0,84 - 0,98). A sensibilidade $\mathrm{e}$ especificidade foram de 100 e $70,3 \%$ respetivamente, para um cut-off de 1,45. Obtivemos para o BEP score uma AUC de $0,81(0,66$ - 0,97) e uma sensibilidade e especificidades de $83 \%$ para um cut-off de 0,06 . (Fig. 2 )

\section{DISCUSSÃO}

Apesar dos avanços técnicos que incluíram a introdução da vacina e a melhoria dos cuidados de estabilização inicial, a DMI continua a ser uma causa de mortalidade e morbilidade em Pediatria. No entanto a mortalidade tem vindo a reduzir na região centro do país, tal como descrito noutros países da Europa. ${ }^{11-13}$ No nosso centro, não ocorrem óbitos por DMI (Fig. 1) desde 2005.

Tabela 2 - Disfunção de órgão e odds ratio ajustado para mortalidade $(n=76)$

\begin{tabular}{lcc}
\hline & $\begin{array}{c}\text { Odds ratio ajustado } \\
(\text { IC }-\mathbf{9 5 \%})\end{array}$ & $\boldsymbol{p}$ \\
\hline Neurológica & 315,0 & $<\mathbf{0 , 0 0 1}$ \\
& $(26,1-3804,1)$ & \\
Renal & 155,0 & $<\mathbf{0 , 0 0 1}$ \\
Respiratória & $(19,5-1299,1)$ & \\
& 53,0 & $<\mathbf{0 , 0 0 1}$ \\
Hematológica & $(6,2-453,9)$ & \\
& 11,7 & $\mathbf{0 , 0 0 6}$ \\
Cardiovascular & $(1,4-96,1)$ & \\
& 0,931 & 0,95 \\
\hline
\end{tabular}




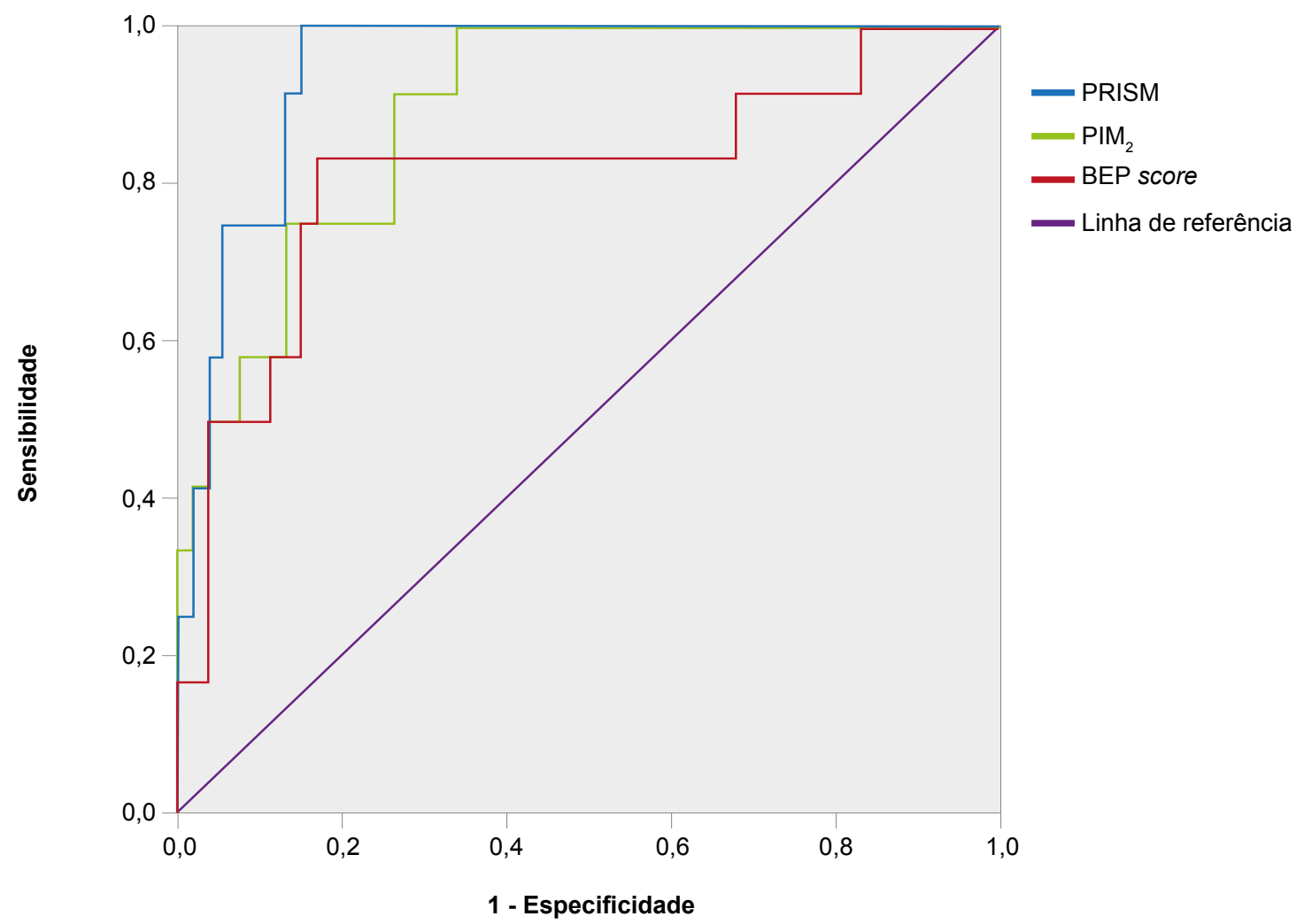

Figura 2 - Curvas ROC para o PRISM (azul), PIM $_{2}$ (verde) e BEP score (vermelho)

A importância dos scores reside na capacidade para avaliar e identificar de forma objetiva doentes mais graves, o que permite facilitar decisões de admissão e orientar as atitudes terapêuticas em cuidados intensivos. Os scores devem possibilitar uma adequada estratificação para otimizar a inclusão de doentes em ensaios clínicos futuros e selecionar aqueles que podem beneficiar de novas terapêuticas. Até aqui estas têm sido aplicadas a grupos heterógenos de doentes, daí, provavelmente, o seu insucesso..$^{14-16}$

A maioria dos scores prognósticos combinam dados clínicos e laboratoriais, outros são apenas baseados em marcadores laboratoriais. Nem todos são específicos para a doença meningocócica e parecem atualmente sobrestimar a mortalidade face à melhoria dos cuidados prestados. ${ }^{17}$

A escala GMSPS foi durante vários anos usada em cuidados intensivos, sendo descrita uma sensibilidade que ronda os $100 \% .{ }^{18,19}$ No entanto, tem como desvantagens o elevado número de variáveis analisadas (sete), e a inclusão de parâmetros subjetivos como a opinião dos pais acerca da evolução da doença.

O PRISM é uma escala genérica usada para comparar o desempenho entre serviços. Avalia o risco de mortalidade nas primeiras 24 horas após admissão em cuidados intensivos. É complexa e requer 14 parâmetros clínicos e analíti$\cos$, incluindo gasometria arterial, coagulação e bioquímica sérica.

$\mathrm{O} \mathrm{PIM}_{2}$ tem a vantagem de poder ser calculado na admissão (primeira hora) mas é demorado e tal como o PRISM necessita de muitas variáveis, sendo também utili- zado para avaliar o desempenho dos serviços.

O produto PN (plaquetas e neutrófilos), que inclui apenas dois parâmetros laboratoriais, baseia-se no grau de resposta inflamatória. Não depende do observador, é rápido e no estudo original parecia ser fidedigno em menores de cinco anos, no entanto apenas foi validado num pequeno número de doentes. ${ }^{17}$

Também o score de Roterdão não tem fatores subjetivos, baseando-se unicamente no excesso de base, plaquetas, potássio e proteína $C$ reativa, que são facilmente obtidos na primeira hora após admissão.

O BEP score necessita apenas de dois valores laboratoriais, de fácil obtenção e que são objetivos.

Mais recentemente foram ainda propostos scores baseados em biomarcadores, no entanto estes não estão facilmente disponíveis na prática clinica diária, estando reservados para estudos de investigação.

No nosso estudo todos os scores de prognóstico analisados mostraram uma boa precisão com sensibilidades e especificidades elevadas, tal como descrito na literatura, apesar da reduzida amostra. ${ }^{3,20} \mathrm{O}$ score que revelou melhor precisão foi o PRISM seguido do $\mathrm{PIM}_{2}$. O BEP score também demonstrou uma boa precisão, apesar de não tão elevada como os outros scores estudados. Contudo, realça-se o facto de o BEP score ser fácil e rapidamente calculado na admissão, a partir de uma fórmula simples, o que contrasta com os outros que necessitam de um maior número de dados e de variáveis, que podem depender do observador. É ainda de salientar que a sua especificidade foi superior à 
Foram limitações deste trabalho a colheita retrospetiva de dados, o que impediu a aplicação do BEP score em todos os doentes, e o reduzido número de casos, que poderia ser colmatado no futuro com a realização de um estudo multicêntrico.

Apesar destas limitações, este estudo contribui para validar a utilização do BEP score na população europeia tal como sugerido numa revisão recente. ${ }^{21}$ Para melhorar a precisão do BEP score e dado que, na nossa análise, uma das disfunções que se associou mais com a mortalidade foi a renal, poderia ser útil acrescentar parâmetros laboratoriais de função renal a este score.

\section{CONCLUSÃO}

Scores de prognóstico genéricos como o PRISM ou o $\mathrm{PIM}_{2}$ mostraram ter uma boa precisão na predição da mortalidade na doença meningocócica invasiva. No entanto são baseados na conjugação de numerosos dados clínicos e laboratoriais, alguns dos quais podem ser de difícil obtenção na admissão em cuidados intensivos.

Apesar de uma precisão não tão elevada comparativamente com os scores supracitados, o BEP score tem a vantagem de ser fácil e rápido de calcular na admissão.

\section{AGRADECIMENTOS}

Os autores agradecem a Margarida Marques do Departamento de Estatística do Centro Hospitalar e Universitário de Coimbra, EPE pela valiosa colaboração prestada na análise estatística.

\section{PROTECÇÃO DE PESSOAS E ANIMAIS}

Os autores declaram que este estudo obedeceu aos princípios éticos para investigação médica em seres humanos consagrados nos regulamentos estabelecidos pelos responsáveis da Comissão de Investigação Clínica e Ética e ainda na Declaração de Helsínquia da Associação Médica Mundial.

\section{CONFIDENCIALIDADE DOS DADOS}

Os autores declaram ter seguido os protocolos do seu centro de trabalho acerca da publicação dos dados de doentes.

\section{CONFLITO DE INTERESSES}

Os autores declaram não ter nenhum conflito de interesses relativamente ao presente artigo.

\section{FONTES DE FINANCIAMENTO}

Não existiram fontes externas de financiamento para a realização deste artigo.

definitions.pdf.

11. Mação $P$, Januário $G$, Ferreira $S$, Dias $A$, Dionísio $T$, Pinto $C$, et al. Doença invasiva meningocócica em cuidados intensivos pediátricos. Acta Med Port. 2014:27:291-4.

12. Maat M, Buysse CM, Emonts M, Spanjaard L, Joosten KF, de Groot R, et al. Improved survival of children with sepsis and purpura: effects of age, gender, and era. Crit Care. 2007;11:R112.

13. Gil-Prieto R, Garcia-Garcia L, Alvaro-Meca A, Gonzalez-Escalada A, Viguera Ester P, Gil De Miguel A. The burden of hospitalizations for meningococcal infection in Spain (1997-2008). Vaccine. 2011;29:576570.

14. Levin M, Quint P, Goldstein B, Barton P, Bradley J, Shemie S, et al. Recombinant bactericidal/permeability increasing protein (rBPI21) as adjunctive treatment for children with severe meningococcal sepsis: a randomized trial. Lancet. 2000;356:961-7.

15. Nadel S, Goldstein B, Williams M, Dalton H, Peters M, Macias WL, et al. Drotrecogin alfa (activated) in children with severe sepsis: a multicenter phase III randomized controlled trial. Lancet. 2007;369:836-43.

16. Holinski $P$, Joff A. A simple meningococcal sepsis prognostic score: focusing on the human animal. Crit Care. 2013;17:172.

17. Peters M, Ross-Russell R, White D, Kerr S, Eaton F, Keengwe I, et al. Early severe neutropenia and thrombocytopenia identifies the highest risk cases of severe meningococcal disease. Ped Crit Care Med. 2001;2:225-31.

18. Thomson A, Sills J, Hart C. Validation of the Glasgow Meningococcal Septicemia Prognostic Score: a 10-year retrospective survey. Crit Care Med. 1991;19:26-30.

19. Shah A, Matthew D. Glasgow Meningococcal Septicemia Prognostic Score in meningococcal septicemia. Crit Care Med. 1992;20:1495-6.

20. Oom P, Rossi R, Correia M, Rodrigues G. Avaliação da gravidade da sépsis meningócica em crianças. Acta Med Port. 2003;16:321-6.

21. Montero-Martín M, Inwald D, Carrol ED, Martinón-Torres F. Prognostic markers of meningococcal disease in children: recente advances and future challenges. Expert Rev Anti Infect Ther. 2014;12:1357-69. 
Luís MARTINS, Patrícia MAÇÃO, Carla PINTO, Teresa DIONÍSIO, Andrea DIAS, Alexandra DINIS, Leonor CARVALHO, José Farela NEVES

\section{Doença Meningocócica Invasiva: Aplicação do Base Excess and Platelets Score numa Unidade de Cuidados Intensivos Pediátricos Portuguesa}

Acta Med Port 2015:28:342-346

Publicado pela Acta Médica Portuguesa, a Revista Científica da Ordem dos Médicos

Av. Almirante Gago Coutinho, 151

1749-084 Lisboa, Portugal.

Tel: +351218428 215

E-mail: submissao@actamedicaportuguesa.com

www.actamedicaportuguesa.com

ISSN:0870-399X | e-ISSN: 1646-0758

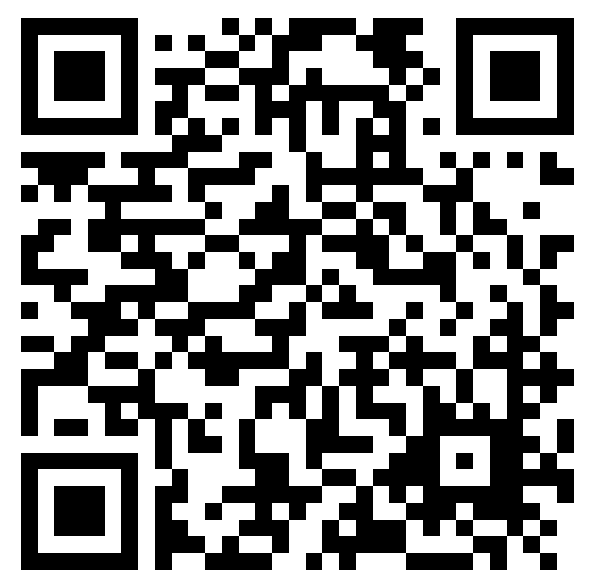

